\title{
Free energy and criticality in the nucleon pair breaking process
}

\author{
M. Guttormsen*, R. Chankova, M. Hjorth-Jensen, J. Rekstad, and S. Siem \\ Department of Physics, University of Oslo, N-0316 Oslo, Norway
}

\author{
A. Schiller \\ Lawrence Livermore National Laboratory, L-414, 7000 East Avenue, Livermore, California 94551, U.S.A. \\ D.J. Dean \\ Physics Division, Oak Ridge National Laboratory, P.O. Box 2008, Oak Ridge, Tennessee 37831, U.S.A.
}

\begin{abstract}
Experimental level densities for ${ }^{171,172} \mathrm{Yb},{ }^{166,167} \mathrm{Er},{ }^{161,162} \mathrm{Dy}$, and ${ }^{148,149} \mathrm{Sm}$ are analyzed within the microcanonical ensemble. In the even isotopes at excitation energies $E<2 \mathrm{MeV}$, the Helmholtz free energy $F$ signals the transition from zero to two quasiparticles. For $E>2 \mathrm{MeV}$, the odd and even isotopes reveal a surprisingly constant $F$ at a critical temperature $T_{c} \sim 0.5 \mathrm{MeV}$, indicating the continuous melting of nucleon Cooper pairs as function of excitation energy.
\end{abstract}

PACS number(s): 21.10.Ma, 24.10.Pa, 25.55.Hp, 27.70.+q

\section{INTRODUCTION}

One of the most spectacular pairing phase transitions in nature is the transition from a normal to a superconducting phase in large electron systems. The transition is triggered at low temperature by massive pairing of two and two electrons into spin $J=0$ pairs, so called Cooper pairs [1].

For atomic nuclei, the pairing phase transition is expected to behave differently. First of all, the nucleus is an isolated, few body system with two species of fermions. Surface effects are prominent, and the coherence length of nucleons coupled in Cooper pairs is larger than the nuclear diameter. Furthermore, there are non-negligible energy spacings between the single particle orbitals. All these facts make the nucleus an inherently small system. Also, other types of residual interactions than pairing are of importance. The influence of these peculiar constraints on the nucleus has been investigated theoretically for a long time [2-5], however, only limited experimental information is available to describe the nature of pairing within the nucleus.

The Oslo group has developed a method to derive simultaneously the level density and $\gamma$-ray strength function from a set of primary $\gamma$-ray spectra [6]. The method has been well tested and today a consistent data set for eight rare earth nuclei is available. In the present work we report for the first time on a comprehensive analysis of the evolution of the pairing phase transition as a function of the nuclear excitation energy.

\section{EXPERIMENTAL LEVEL DENSITIES}

Level densities for ${ }^{171,172} \mathrm{Yb},{ }^{166,167} \mathrm{Er},{ }^{161,162} \mathrm{Dy}$, and ${ }^{148,149} \mathrm{Sm}$ have been extracted from particle- $\gamma$ coincidences. The experiments were carried out with $45 \mathrm{MeV}$ ${ }^{3} \mathrm{He}$ projectiles accelerated by the MC-35 cyclotron at the University of Oslo. The data were recorded with the CACTUS multidetector array using the pick up $\left({ }^{3} \mathrm{He}, \alpha \gamma\right)$ reaction on ${ }^{172,173} \mathrm{Yb},{ }^{167} \mathrm{Er},{ }^{162,163} \mathrm{Dy}$, and ${ }^{149} \mathrm{Sm}$ targets and the inelastic $\left({ }^{3} \mathrm{He},{ }^{3} \mathrm{He} \gamma \gamma\right)$ reaction on ${ }^{167} \mathrm{Er}$ and ${ }^{149} \mathrm{Sm}$ targets. The charged ejectiles were detected with eight $\Delta E-E$ particle telescopes placed at an angle of $45^{\circ}$ relative to the beam direction. Each telescope comprises one $\mathrm{Si}$ front and one $\mathrm{Si}(\mathrm{Li})$ back detector with thicknesses of 140 and $3000 \mu \mathrm{m}$, respectively. An array of $28 \mathrm{NaI} \gamma$ ray detectors with a total efficiency of $\sim 15 \%$ surrounds the target and particle detectors. From the reaction kinematics, the measured ejectile energy can be transformed into excitation energy $E$. Thus, each coincident $\gamma$ ray can be assigned to a $\gamma$ cascade originating from a specific energy $E$. These spectra are the basis for the extraction of level density and $\gamma$-strength function as described in Ref. [6]. Several interesting applications of the method have been demonstrated, see, e.g., Refs. [7-10].

The level densities for ${ }^{171,172} \mathrm{Yb},{ }^{166,167} \mathrm{Er},{ }^{161,162} \mathrm{Dy}$, and ${ }^{148,149} \mathrm{Sm}$ are shown in Fig. 1 . The level densities are normalized at low excitation energies where (almost) all levels are known, and at the neutron binding energy $B_{n}$ where the level density can be estimated from neutronresonance spacings. The spin window populated in the reactions is typically $I \sim 2-6 \hbar$. Already, three general comments can be made to these data: (i) above $2 \mathrm{MeV}$ excitation energy, all level densities are very linear in a log plot, suggesting a so-called constant temperature

*Electronic address: magne.guttormsen@fys.uio.no 
level density, (ii) the level densities of the odd-even isotopes are larger than for their neighboring even-even isotopes, and (iii) the even-even isotopes show a strong increase in level density between 1 and $2 \mathrm{MeV}$, indicating the breaking of Cooper pairs.

It should be noted that the transitions considered here are low temperature phenomena. The ${ }^{171,172} \mathrm{Yb}$, ${ }^{166,167} \mathrm{Er}$, and ${ }^{161,162}$ Dy nuclei have well deformed shapes, and various calculations in this mass region [11-14] indicate that the transition from deformed to spherical shape occurs at much higher temperatures than the temperature at which the first pairs break. However, for nuclei closer to the $N=82$ shell gap, e.g., ${ }^{148,149} \mathrm{Sm}$, the coexistence between deformed and spherical shapes at low temperatures cannot be excluded, as discussed in Ref. [15].

\section{FREE ENERGY AND CRITICAL TEMPERATURE}

The statistical microcanonical ensemble is an appropriate working frame for describing an isolated system like the nucleus. In this ensemble the excitation energy $E$ is fixed, in accordance with the observables of our experiments. The microcanonical entropy is given by the number of levels $\Omega$ at $E$

$$
S(E)=k_{B} \ln \Omega(E),
$$

where the multiplicity $\Omega$ is directly proportional to the level density $\rho$ by $\Omega(E)=\rho(E) / \rho_{0}$. The normalization denominator $\rho_{0}$ is adjusted to give $S \sim 0$ for $T \sim 0$, which fulfills the third law of thermodynamics. Here, we assume that the lowest levels of the ground state bands of the ${ }^{172} \mathrm{Yb},{ }^{166} \mathrm{Er},{ }^{162} \mathrm{Dy}$, and ${ }^{148} \mathrm{Sm}$ nuclei have temperatures close to zero, giving on the average $\rho_{0}=2.2 \mathrm{MeV}^{-1}$. In the following, this value is used for all eight nuclei and Boltzmann's constant is set to unity $\left(k_{B}=1\right)$.

In order to analyze the criticality of low temperature transitions, we investigate the probability $P$ of a system at the fixed temperature $T$ to have the excitation energy $E$, i.e.,

$$
P(E, T, L)=\Omega(E) \exp (-E / T) / Z(T),
$$

where the canonical partition function is given by $Z(T)=$ $\int_{0}^{\infty} \Omega\left(E^{\prime}\right) \exp \left(-E^{\prime} / T\right) d E^{\prime}$. Implicitly, the multiplicity of states $\Omega(E)$ depends on the size of the system, denoted by $L$. Often, it is more practical to use the negative logarithm of this probability $A(E, T)=-\ln P(E, T)$, where we in the following omit the $L$ parameter. Lee and Kosterlitz showed $[16,17]$ that the function $A(E, T)$, for a fixed temperature $T$ in the vicinity of a critical temperature $T_{c}$ of a structural transition, will exhibit a characteristic double-minimum structure at energies $E_{1}$ and $E_{2}$. For the critical temperature $T_{c}$, one finds $A\left(E_{1}, T_{c}\right)=A\left(E_{2}, T_{c}\right)$. It can be easily shown that $A$ is closely connected to the Helmholtz free energy and the previous condition is equivalent to

$$
F_{c}\left(E_{1}\right)=F_{c}\left(E_{2}\right)
$$

a condition which can be evaluated directly from our experimental data. Here, it should be emphasized that $F_{c}$ is a linearized approximation to the Helmholtz free energy at the critical temperature $T_{c}$ according to $F_{c}(E)=$ $E-T_{c} S(E)$, thereby avoiding the introduction of a caloric curve $T(E)$. The free-energy barrier at the intermediate energy $E_{m}$ between $E_{1}$ and $E_{2}$, is given by

$$
\Delta F_{c}=F_{c}\left(E_{m}\right)-F_{c}\left(E_{1}\right)
$$

Now, the evolution of $\Delta F_{c}$ with increasing system size $L$ may determine the order of a possible phase transition $[16,17]$. These ideas have, e.g., recently been applied to analyze phase transitions in a schematic pairing model [18].

Figure 2 displays a schematic description of the entropy for even-even, odd-mass and odd-odd nuclei as function of excitation energy. In the lower excitation energy region of the even-even nucleus, only the ground state is present, and above $E \sim 2 \Delta$ the level density is assumed to follow a constant temperature formula. It has been shown $[19,20]$ that the single particle entropy $s$ is an approximately extensive thermodynamical quantity in nuclei at these temperatures. The increase in entropy at the breaking of the first proton or neutron pair, i.e., at $E=2 \Delta$, is roughly $2 s$ in total for the two newly created unpaired nucleons. The requirement $F_{c}\left(E_{1}\right)=F_{c}\left(E_{2}\right)$, where $T=T_{c}$, gives $E_{2}-E_{1}=T_{c}\left[S\left(E_{2}\right)-S\left(E_{1}\right)\right]$. Thus, with the assumed estimates above, we obtain the relation $\Delta=T_{c} s$, which may be used to extract the critical temperature for the pairing transition. Adopting typical values of $\Delta=1 \mathrm{MeV}$ and $s=2 k_{B}$ [19,20], we obtain $T_{c}=0.5 \mathrm{MeV}$. For the odd-mass case, one starts out with one quasiparticle which gives roughly one unit of single-particle entropy $s$ around the ground state. The three quasiparticle regime appears roughly at $E=2 \Delta$ with a total entropy of $3 \mathrm{~s}$. The region between $E=0$ and $2 \mathrm{MeV}$ is modeled with a step at $\sim 2 \mathrm{MeV}$, however, in real nuclei the level density is almost linear in a log-plot for the whole excitation energy region due to the smearing effects of the valence nucleon. In the case of an odd-odd nucleus, one starts out with two units of singleparticle entropy. The two valence nucleons represent a strong smearing effect on the level density and the modeled step structure in entropy at $E=2 \Delta$ for the onset of the four quasiparticle regime is completely washed out.

In the higher excitation region, further steps for transitions to higher quasiparticle regimes are also washed out due to the strong smearing effects of the already present, unpaired nucleons. The slope of the entropy with excitation energy is determined by two competing effects: the quenching of pairing correlations which drives the cost in energy lower for the breaking of additional pairs, and the Pauli blocking which reduces the entropy created per 
additional broken pair. The competing influence of both effects is modeled by a constant-temperature level density with the same slope for all three nuclear systems and a slightly higher critical temperature. In this region of the model, there are infinitely many excitation energies where the relation $F_{c}\left(E_{1}\right)=F_{c}\left(E_{2}\right)$ is fulfilled.

The breaking of proton or neutron pairs are thought to take place at similar excitation energies due to the approximate isospin symmetry of the strong interaction. It is indeed commonly believed that the pairing gap parameter $\Delta$ and, thus, the critical temperature $T_{c}$ for the breaking of Cooper pairs, are approximately the same for protons and neutrons. Furthermore, interactions between protons and neutrons will certainly wash out any differences in behavior between the proton and neutron fluids. In Fig. 3, the influence of differences in proton and neutron pair breaking is investigated within our schematic model. Here, we assume that neutrons break up at $2 \Delta$ creating an entropy of $2 s$. The protons are assumed to break up at $10 \%$ higher excitation energy (since $Z<N$ ) creating $10 \%$ less entropy (due to the larger proton single particle level spacing). The entropy of the total system of either proton or neutron pair breaking gives

$$
S(E)=\ln \left[e^{S_{p}(E)}+e^{S_{n}(E)}\right]-\ln 2,
$$

where the last term assures that $S=0$ in the ground state band. The requirement $F_{c}\left(E_{1}\right)=F_{c}\left(E_{2}\right)$ gives $T_{c}^{(n)}=\Delta / s$ for neutrons (as in Fig. 2) and $T_{c}^{(p)}=$ $1.1 \Delta / 0.9 \mathrm{~s}=1.22 \Delta / \mathrm{s}$ for protons. In the combined system of both neutrons and protons, a value of $T_{c}=$ $\left(T_{c}^{(n)}+T_{c}^{(p)}\right) / 2=1.11 \Delta / s$ is deduced from Fig. 3. Thus, typical fluctuations in the pairing gap parameter and the single particle entropy for neutrons and protons give only small changes in the extracted critical temperature.

\section{EXPERIMENTAL RESULTS}

In order to experimentally investigate the behavior for even isotopes, linearized free energies $F_{c}$ for certain temperatures $T_{c}$ are displayed in Fig. 4. The data clearly reveal two minima with $F_{c}\left(E_{1}\right)=F_{c}\left(E_{2}\right)=F_{0}$, which is due to the general increase in level density around $E \sim 2 \mathrm{MeV}$, as schematically shown in Fig. 2. For all nuclei, we obtain $E_{1} \sim 0$ and $E_{2} \sim 2 \mathrm{MeV}$ which compares well with $2 \Delta$. We interpret the results of Fig. 4 as the transition due to the breaking of the very first nucleon pairs. The deduced critical temperatures are $T_{c}=0.47$, 0.40, 0.47 and $0.45 \mathrm{MeV}$ for ${ }^{172} \mathrm{Yb},{ }^{166} \mathrm{Er},{ }^{162} \mathrm{Dy}$, and ${ }^{148} \mathrm{Sm}$, respectively.

Recently [7], another method was introduced to determine the critical temperatures in the canonical ensemble. Here, the constant temperature level density formula for the canonical heat capacity $C_{V}(T)=(1-T / \tau)^{-2}$ was fitted to the data in the temperature region of $0-0.4 \mathrm{MeV}$ corresponding to excitation energies between $0-2 \mathrm{MeV}$, and the fitted temperature parameter $\tau$ was then identified with the critical temperature $T_{c}$. Since a constant temperature level density formula implies a constant linearized Helmholtz free energy $F_{c}(E)$ (provided $\tau=T_{c}$ ), this former method is almost equivalent to the present method, i.e., of identifying the temperature $T_{c}$ for which the linearized Helmholtz free energy is in average constant. Therefore, it is not surprising that the extracted critical temperatures $T_{c}=0.49,0.44,0.49$ and $0.45 \mathrm{MeV}$ for the respective nuclei using the older method $[7,8,10]$ coincide well with the critical temperatures presented in this work. However, while the previous method was based on an ad-hoc assumption of the applicability of a constant temperature level density formula, the present method has a much firmer theoretical foundation.

The height of the free-energy barrier should show a different dependence on the system size $L$ according to the order of a possible phase transition [16,17]. The barriers deduced from Fig. 4 yield $\Delta F_{c} \sim 0.5-0.6 \mathrm{MeV}$, values which seem not to have any systematic dependence on the mass number $A$ within the experimental uncertainties. Even with better data, an unambiguous dependence of the barrier height on the system size would be unlikely when using $A$ as a measure for the parameter $L$ since the relevant system size for the very first breaking of Cooper pairs might be characterized by only a few valence nucleons. Another complicating interference is that other properties of the nuclear system which might influence the onset of pair breaking also change with mass number, e.g., deformation, pairing gap, and locations of single particle levels around the Fermi surface.

In the schematic model of Fig. 2, we would expect a free energy barrier of $\Delta F_{c}=2 \Delta \sim 2 \mathrm{MeV}$ at $E=$ $2 \Delta \sim 2 \mathrm{MeV}$. However, the data are more consistent with the dotted lines of Fig. 2 indicating a smoother behavior around the expected steps due to the existence of collective excitations like rotation and $\beta, \gamma$, and octupole vibrations between 1 and $2 \mathrm{MeV}$ for the even-even nuclei, and due to the increasing availability of single-particle orbitals for the odd nucleon in the case of odd nuclei. Thus, we expect the centroid of the barrier to be shifted down in energy with a corresponding proportional reduction of the barrier height, and an inspection of Fig. 4 indeed shows that the free energy barrier is $0.5-0.6 \mathrm{MeV}$ at $\sim 0.6 \mathrm{MeV}$ excitation energy for the even-even nuclei. A similar analysis of the odd isotopes is difficult to accomplish since there seems not to be any common structures. Here, the unpaired valence neutron smears out the effects of the depairing process too much to be visible in the present data. However, it has been attempted in Ref. [8] to interpret the structure in the level density of ${ }^{167} \mathrm{Er}$ around $1 \mathrm{MeV}$ in terms of a first order phase transition.

The smearing effect is expected to be even more pronounced for the breaking of additional pairs. Figure 5 shows the linearized Helmholtz free energy for all eight nuclei investigated, but at slightly higher critical temperatures than in Fig. 4. The critical temperature $T_{c}$ is found by a least $\chi^{2}$ fit of a constant value $F_{0}$ to the 
experimental data. The fit region is from $E=2 \mathrm{MeV}$ up to 5 and $7 \mathrm{MeV}$ for the odd and even isotopes, respectively, giving normalized $\chi^{2}$ values in the range from 0.5 to 2.5. Here, instead of a double-minimum structure, a continuous "minimum" of $F_{c}$ is displayed for several MeV. This observation allows us to conclude that the further depairing process cannot under any circumstances be interpreted as an abrupt structural change in the nucleus typical for a first order phase transition. The constant lines of Fig. 5 visualize how surprisingly well $F_{0}$ fits the data: the deviations are typically less than $100 \mathrm{keV} .{ }^{1}$ The ongoing breaking of further Cooper pairs overlapping in excitation energies above $2 \mathrm{MeV}$ is therefore contrary to what is found in the schematic model of Ref. [18]. This is probably due to strong residual interactions in real nuclei, like the quadrupole-quadrupole interaction, which were not taken into account in the model calculation. Thus, (nearly) all excitation energies above $2 \mathrm{MeV}$ will energetically match with the costs of breaking nucleon pairs. Here, all excess energy goes to the process of breaking pairs. Since the gain in entropy $d S$ is proportional to $d E$, the microcanonical temperature, $T(E)=(d S / d E)^{-1}$, remains constant as function of excitation energy, and the level density displays a straight section in the log plot.

At higher excitation energies than measured here, the pairing correlations vanish and the system behaves more like a Fermi gas. Here, the free energy will indicate the closing stage of the depairing process by increasing $F_{c}$, with $F_{c}>F_{0}$. However, in this regime also shape transitions and fluctuations as well as the melting of the shell structure may play a role and give deviations from a simple Fermi gas model with $\rho \propto \exp (2 \sqrt{a E}), a$ being the level density parameter. Unfortunately, these very interesting phenomena cannot be investigated with the present experimental data.

The fitted value $F_{0}$ contains information on the entropy of the system at $T_{c}$ through $S=\left(E-F_{0}\right) / T_{c}$. In Fig. 6, we have compared the entropy for the various nuclei at an excitation energy $E=4 \mathrm{MeV}$, an energy where all nuclei seem to "behave" equally well (see Fig. 5 ). Figure 6 also shows that the odd mass nuclei display generally higher entropy regardless of the mass number $A$ being one higher or lower than the neighboring even isotope. We also observe that since the ${ }^{148,149} \mathrm{Sm}$ nuclei are not mid-shell nuclei, they show less entropy, reflecting the lower single particle level density when approaching the $N=82$ shell gap. By evaluating the odd-even $\delta S=S_{\text {odd }}-S_{\text {even }}$, we find $\delta S \sim 2$ for all four isotopes, as shown in the lower panel of Fig. 6. This means that excited holes and particles have the same degree of freedom with respect to the even mass nuclei.

\section{CONCLUSION}

Unique experimental information on level densities for eight rare earth nuclei is utilized to extract thermodynamic quantities in the microcanonical ensemble. The linearized Helmholtz free energy is used to obtain the critical temperatures of the depairing process. For a critical temperature just below $T_{c} \sim 0.5 \mathrm{MeV}$, we observe a structural transition of even nuclei in the $E=0-2 \mathrm{MeV}$ region due to the breaking of the first nucleon pair. Unfortunately, it was not possible to use the development of the barrier height $\Delta F_{c}$ with the size of the system $L$ to conclude on the presence of a thermodynamical phase transition and its order. The critical temperature for the melting of other pairs is found at slightly higher temperatures. Here, we obtain a surprisingly constant value for the linearized Helmholtz free energy, indicating a continuous melting of nucleon Cooper pairs as function of excitation energy. The conspicuous absence of a doubleminimum structure in $F_{c}$ for this process is at variance with the presence of a first-order phase transition in the thermodynamical sense. The entropy difference between odd and even systems is found to be constant with respect to excitation energy and is consistent with the expected values of the single particle entropy in these nuclei.

\section{ACKNOWLEDGMENTS}

Financial support from the Norwegian Research Council (NFR) is gratefully acknowledged. Part of this work was performed under the auspices of the U.S. Department of Energy by the University of California, Lawrence Livermore National Laboratory under Contract No. W7405-ENG-48. Research at Oak Ridge National Laboratory was sponsored by the Division of Nuclear Physics, U.S. Department of Energy under contract DE-AC0500OR22725 with UT-Battelle, LLC.

[1] J. Bardeen, L.N. Cooper, and J.R. Schrieffer, Phys. Rev. 108, 1175 (1957).

[2] Mitsuo Sano and Shuichiro Yamasaki, Prog. Theor. Phys. 29, 397 (1963).

[3] L.G. Moretto, Nucl. Phys. A185, 145 (1972).

[4] K. Tanabe and K. Sugawara-Tanabe, Phys. Lett. 97B, 337 (1980).

\footnotetext{
${ }^{1}$ This fact might also settle the discussion in Ref. [8] and discard the possible interpretation of the many negative branches of the microcanonical heat capacity observed in Fig. 8 of this reference as indicators of separate first-order phase transitions.
} 
[5] Alan L. Goodman, Nucl. Phys. A352, 45 (1981).

[6] A. Schiller, L. Bergholt, M. Guttormsen, E. Melby, J. Rekstad, and S. Siem, Nucl. Instrum. Methods Phys. Res. A447, 498 (2000).

[7] A. Schiller, A. Bjerve, M. Guttormsen, M. Hjorth-Jensen, F. Ingebretsen, E. Melby, S. Messelt, J. Rekstad, S. Siem, and S.W. Ødegård, Phys. Rev. C 63, 021306 (2001).

[8] E. Melby, M. Guttormsen, J. Rekstad, A. Schiller, S. Siem, and A. Voinov, Phys. Rev. C 63, 044309 (2001).

[9] A. Voinov, M. Guttormsen, E. Melby, J. Rekstad, A. Schiller, and S. Siem, Phys. Rev. C 63, 044313 (2001).

[10] S. Siem, M. Guttormsen, K. Ingeberg, E. Melby, J. Rekstad, A. Schiller, and A. Voinov, Phys. Rev. C 65, 044318 (2002).

[11] D.J. Dean, S.E. Koonin, G.H. Lang, P.B. Radha, and W.E. Ormand, Phys. Lett. B 317, 275 (1993).

[12] J.A. White, S.E. Koonin, and D.J. Dean, Phys. Rev. C 61, 034303 (2000).

[13] J.L. Egido, L.M. Robledo, and V. Martin, Phys. Rev.
Lett. 85, 26 (2000).

[14] B.K. Agrawal, Tapas Sil, S.K. Samaddar, and J.N. De, Phys. Rev. C 63, 024002 (2001).

[15] N.V. Zamfir, R.F. Casten, M.A. Caprio, C.W. Beausang, R. Krücken, J.R. Novak, J.R. Cooper, G. Cata-Danil, and C.J. Barton, Phys. Rev. C 60, 054312 (1999), and references therein.

[16] Jooyoung Lee and J.M. Kosterlitz, Phys. Rev. Lett. 65, 137 (1990)

[17] Jooyoung Lee and J.M. Kosterlitz, Phys. Rev. B 43, 3265 (1991).

[18] A. Belić, D.J. Dean, and M. Hjorth-Jensen, preprint, cond-mat/0104138 (2001).

[19] M. Guttormsen, A. Bjerve, M. Hjorth-Jensen, E. Melby, J. Rekstad, A. Schiller, S. Siem, and A. Belić, Phys. Rev. C 62, 024306 (2000).

[20] M. Guttormsen, M. Hjorth-Jensen, E. Melby, J. Rekstad, A. Schiller, and S. Siem, Phys. Rev. C 63, 044301 (2001). 


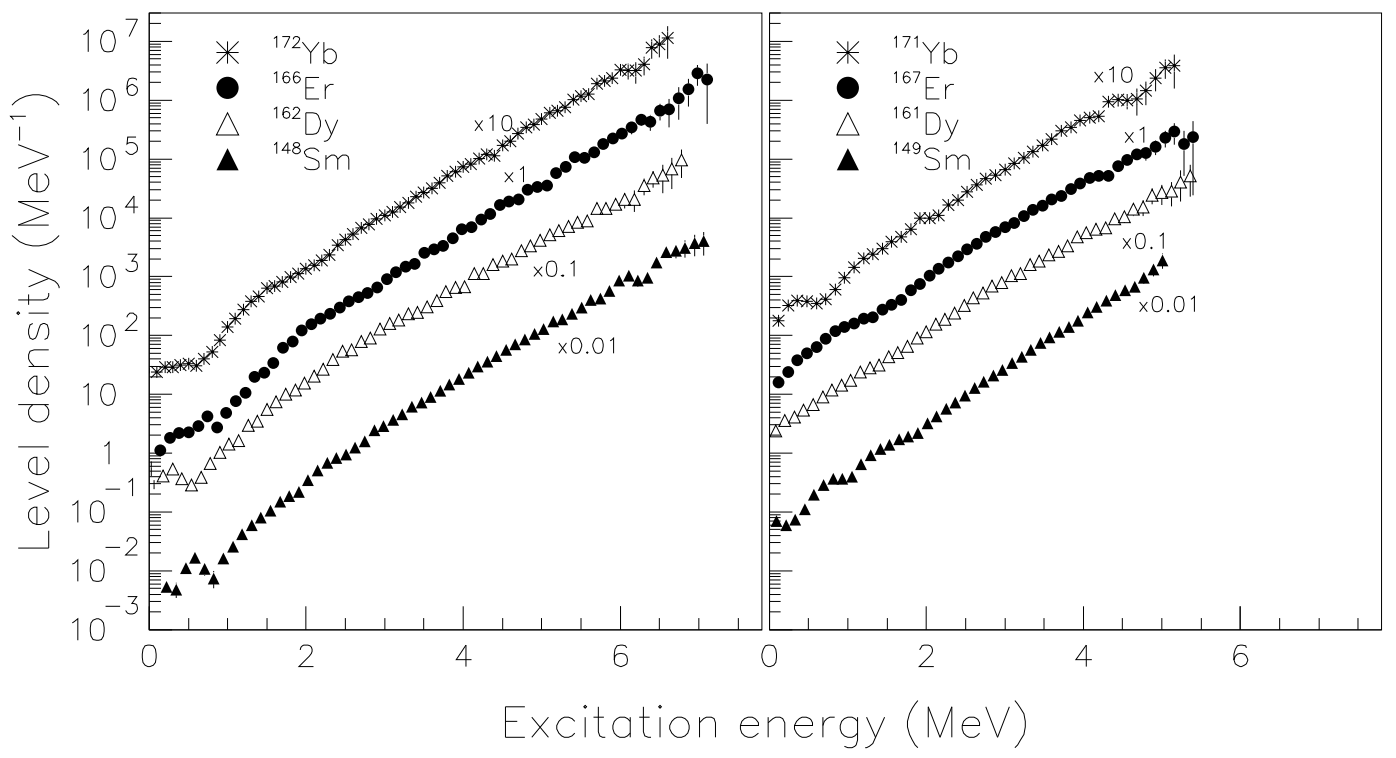

FIG. 1. Experimental level densities for the nuclei ${ }^{171,172} \mathrm{Yb},{ }^{166,167} \mathrm{Er},{ }^{161,162} \mathrm{Dy}$, and ${ }^{148,149}$ Sm. The data are taken from Refs. $[7,8,10]$. 

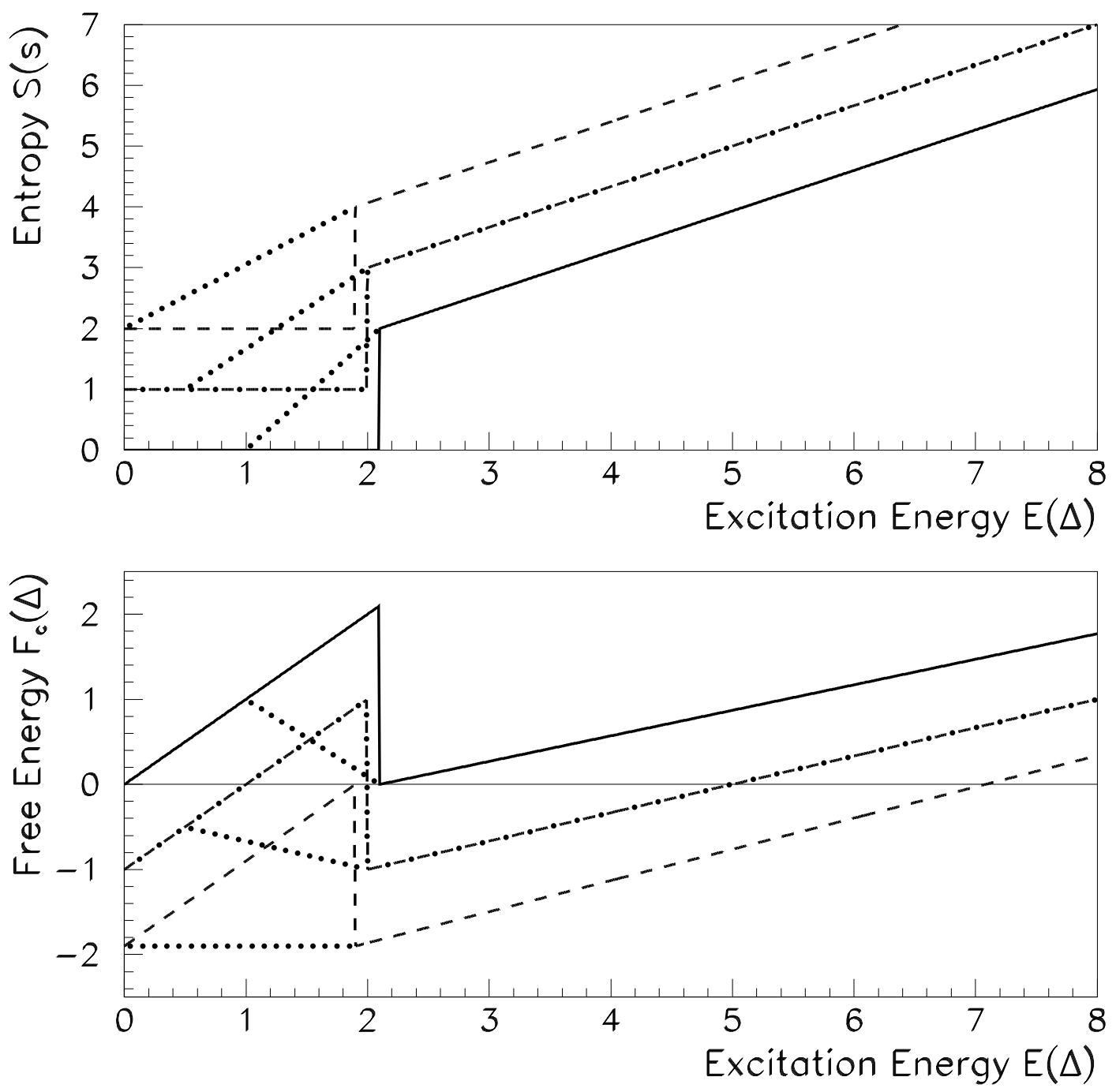

FIG. 2. Schematic representation of the entropy $S$ in units of the single particle entropy $s$ (top panel) for even-even (solid line) odd-mass (dash-dotted line) and odd-odd (dashed line) nuclei. For the purpose of the figure, the steps in entropy are drawn slightly staggered in energy. Lower panel: linearized Helmholtz free energy $F_{c}$ at the critical temperature $T_{c}$ of even-even, odd-mass and odd-odd nuclei. All energies are measured in units of the pairing gap parameter $\Delta$. The dotted lines indicate the situation if additional levels are included below the steps in entropy. 

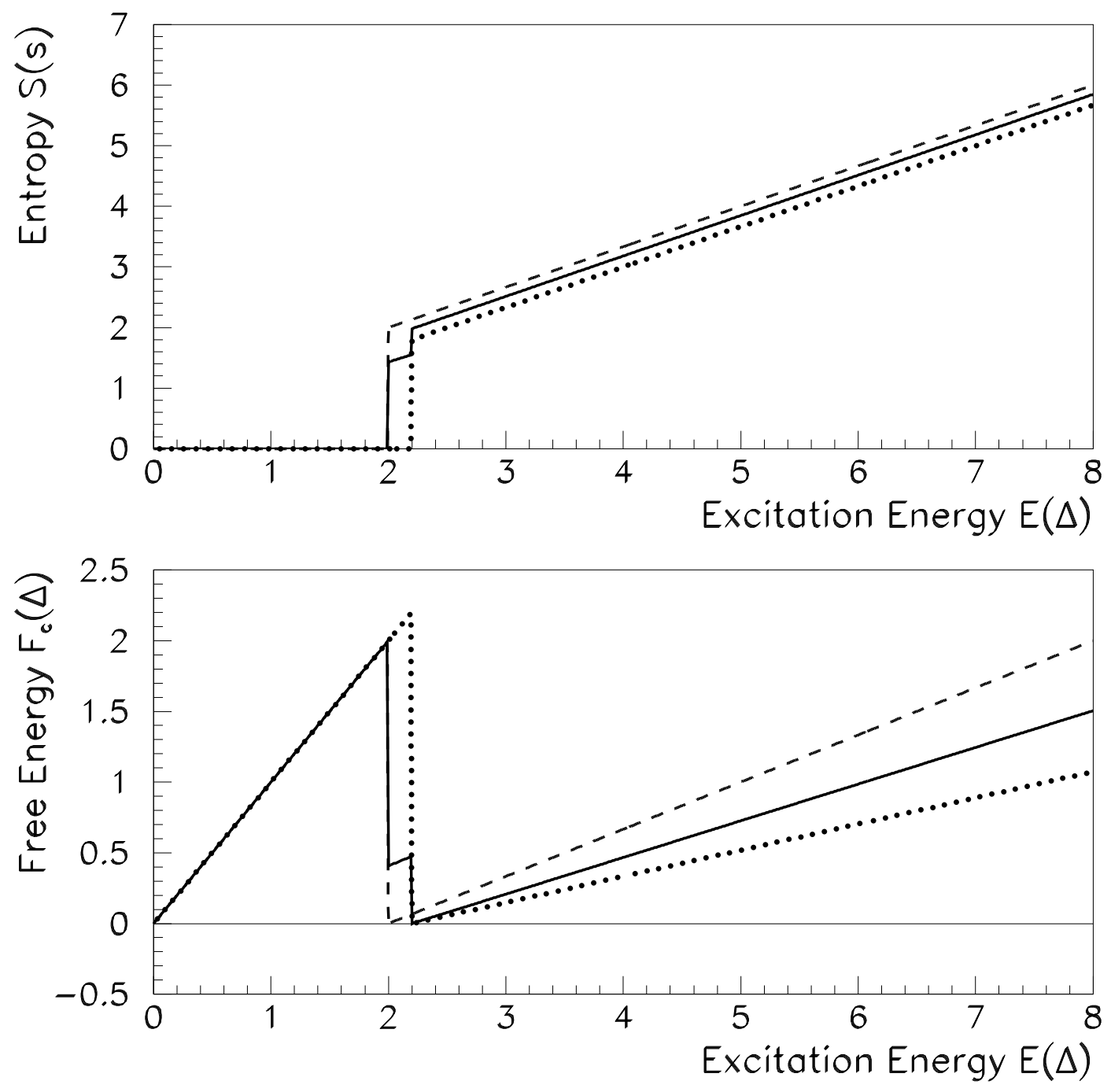

FIG. 3. Same as previous figure in the case of an even-even nucleus but for unequal proton and neutron fluids. Curves are given for the neutron fluid alone (dashed lines with pairing gap parameter $\Delta$ and single particle entropy $s$ ), the proton fluid alone (dotted lines with $1.1 \Delta$ and $0.9 s$ ) and the composite system (solid lines). 


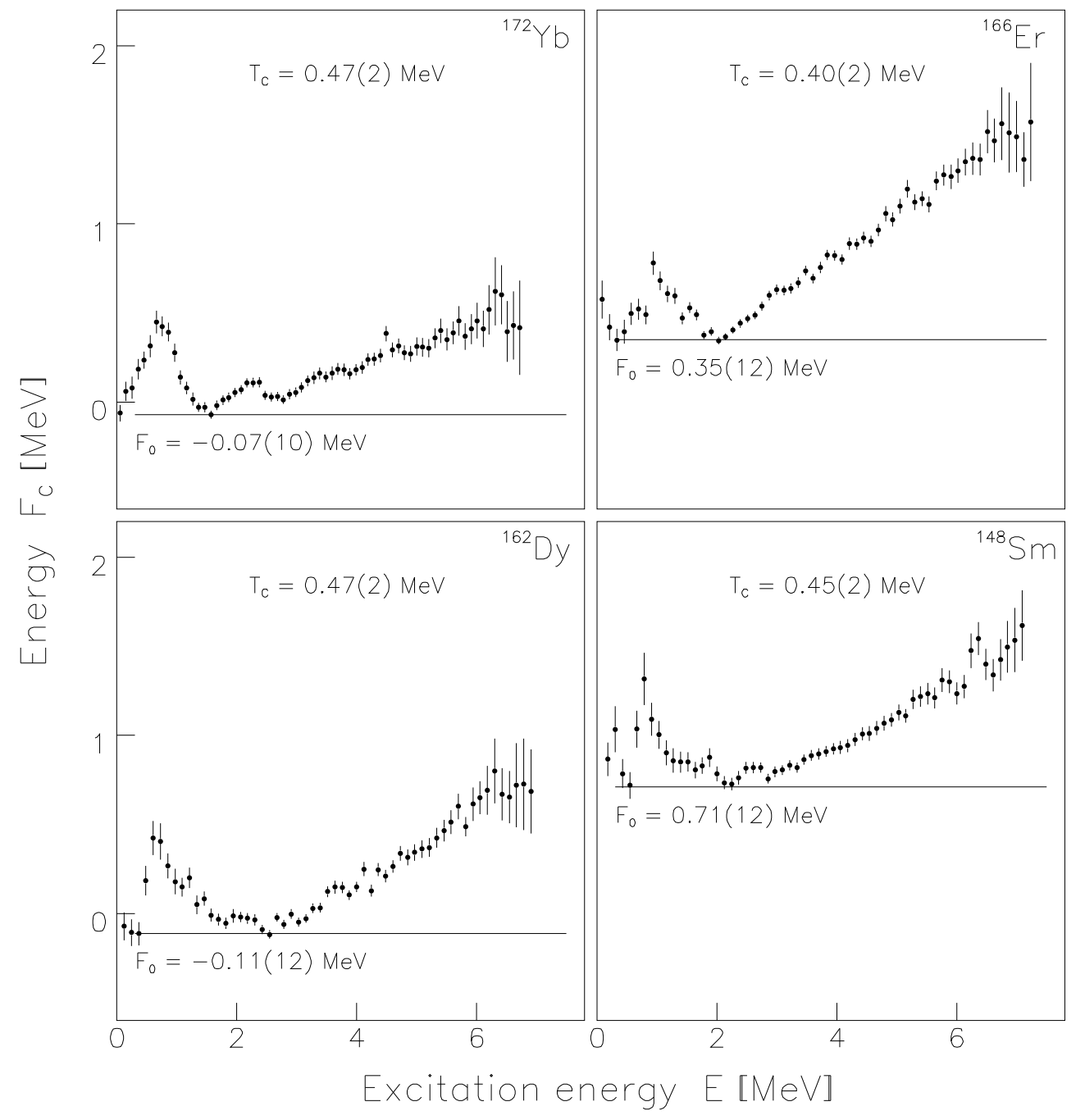

FIG. 4. Linearized Helmholtz free energy at the critical temperature $T_{c}$. The constant level $F_{0}$ connecting the two minima is indicated by lines. 


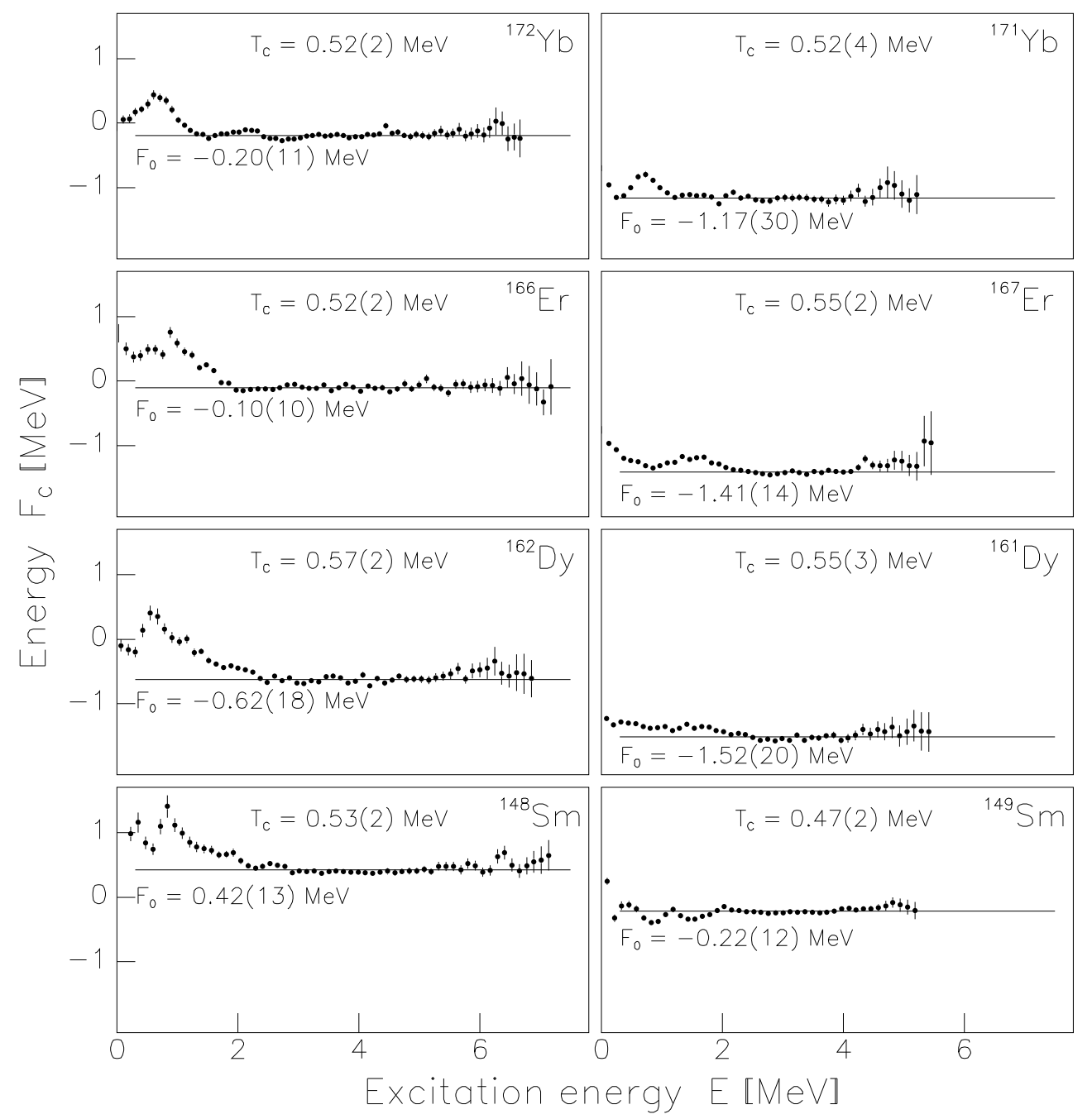

FIG. 5. Linearized Helmholtz free energy at the critical temperature $T_{c}$. The fitted constant level $F_{0}$ is indicated by lines. 


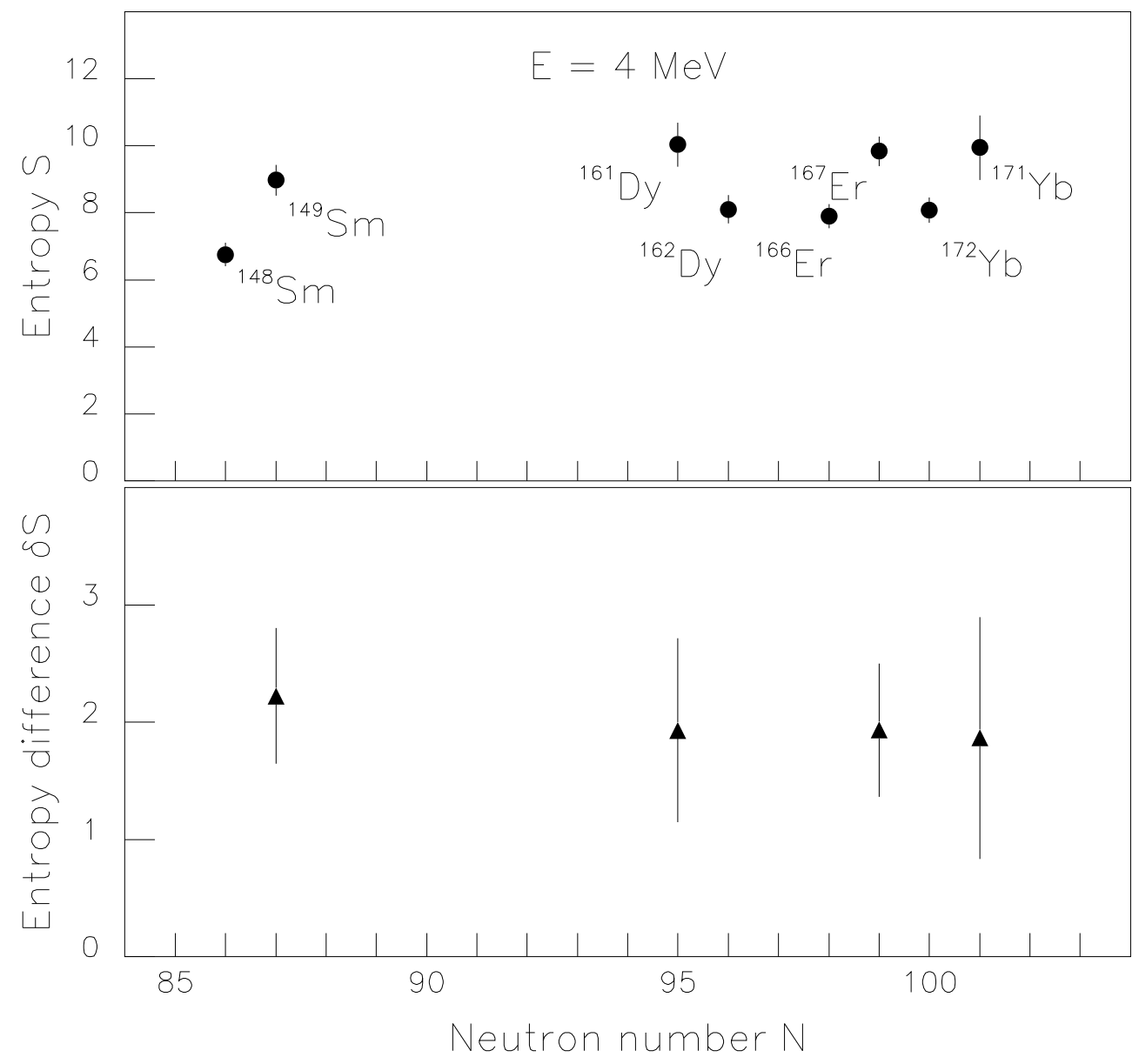

FIG. 6. Experimental entropy evaluated in the microcanonical ensemble at excitation energy $E=4 \mathrm{MeV}$ and temperature $T_{c}$. In the lower panel the odd-even difference $\delta S=S_{\text {odd }}-S_{\text {even }}$ is displayed for the four isotopes. 